\title{
Kommunikationsgerechte Pädagogik der „Totalen Sprache”: ein Entwurf
}

\author{
von Francisco Gutiérrez Pérez
}

\section{Eine auf die Kommunikationsmittel gegründete Pädagogik}

\section{A. Die Gründe für eine Pädagogik der Totalen Sprache}

Vieles hat sich seit der Erfindung und dem ersten Einsatz der Druckpresse geändert. Wenn auch das Mittelalter noch in Verhaltensweisen und Begriffen, in besonderen Lebensformen, bis in die fortgeschrittensten Gesellschaften hinein vorherrscht, so hat es doch als soziale, politische und wirtschaftliche Struktur an Boden verloren. Ebenso weichen die Monarchien und auch die bürgerlichen Gesellschaften vor den sozialistischen Gesellschaften zurück. Es werden gemeinschaftliche Lebensformen erprobt, in denen kein Mensch sich dem anderen aufdrängt. Das Bezugsfeld zwischen den gesellschaftlichen Strukturelementen macht heutzutage wesentliche Veränderungen durch.

Der wissenschaftliche Fortschritt hat uns mit uns selbst konfrontiert sowie mit dem gesamten Universum, mit unendlichen Möglichkeiten des Forschens und Wissens; er fördert unentwegt die Entwicklung von Techniken, die uns die von den Utopisten erdachte Welt erschreckend gegenwärtig rücken: sie kommt uns bereits zum Greifen nahe.

Doch zugleich erweisen sich Wissenschaft und Technik wie auch die neuen gesellschaftlichen Organisationen als widerspruchsvoll und schaffen ehedem unbekannte Probleme: unter anderem die Erziehungskrise.

Innerhalb dieser Krise ist wohl ein wichtiger Aspekt der geringe oder schlechte Gebrauch, den man von sämtlichen heute vorhandenen Kommunikationstechniken macht, wie auch der Konflikt, den das Suchen nach gemeinschaftlichen Lebensformen unter den Menschen gegenüber einer paternalistischen, straff hierarchisch geführten Erziehung auslöst: der Schüler ist dem Lehrer untergeordnet, der Lehrer den Aufsichtsbeamten und Direktoren, und so bilden sie eine Pyramide. Dieses mittelalterliche Strukturrelikt rückt die Planer der Erziehung nicht nur von den Schülern, sondern auch von der Wirklichkeit $a b$, die nicht einheitlich, sondern variabel, plural und heterogen geprägt ist.

Die Erziehung muß aus den Gemeinschaften selbst erwachsen. Sowohl Lehrer wie Schüler haben Weggefährten einer gemeinsamen Erfahrung und Suche zu sein. Auf diese wechselseitige Bezogenheit untereinander gründet die Pädagogik der Totalen Sprache ihr Existenzrecht.

Die Schule, die sich der traditionellen Methoden bedient, wird die von der gegenwärtigen Gesellschaft geforderte Zweckbestimmung nicht erreichen können.

Francisco Gutiérrez Pérez ist Direktor des Lateinamerikanischen Zentrums für "Totale Sprache" in Peru. Sein Beitrag ist von Erfahrungen in Lateinamerika diktiert. Begründer und Vertreter der "langue totale“ in Frankreich ist P. Antoine Vallet. 
Nur mit der unmittelbar zugreifenden Unterrichtung einer jeden Ausdrucksform der gegenwärtigen Gesellschaft geht die Pädagogik die ernsten Probleme an, die die Welt von heute kennzeichnen.

Der Mensch muß innerhalb seiner eigenen Wirklichkeit erzogen werden. Dazu hat die Schule dem zu Erziehenden die Möglichkeiten anzubieten, damit er zu seiner eigenpersönlichen Verwirklichung gelangt.

Die Pädagogik der Totalen Sprache vermittelt dem zu Erziehenden Möglichkeiten, sich mit der ganzen Fülle und Dynamik seines Seins auszudrücken.

Trotz der in das Schulsystem eingebrachten Erziehungsreformen bietet die heutige Schule dem zeitgenössischen Menschen keine Antwort auf seine Schwierigkeit, sich in einer bestimmten gesellschaftlichen Umwelt als Mensch zu verwirklichen.

Der Mensch der Bild-Kultur muß dazu erzogen werden, diese Sprache, die sowohl die seine wie auch die seiner Zeit ist, voll $\mathrm{zu}$ verstehen. Er muß so heranwachsen, daß er selbst in seinem Leben jene Unruhe weckt, die den tiefen Bedürfnissen seines Menschseins gerecht wird.

Die Pädagogik der Totalen Sprache, als Bewußtmachung der modernen Sprache, der Sprache des 20. Jahrhunderts, muß diese erzieherische und kulturelle Synthese zuwegebringen. Sie muß innerhalb wie außerhalb des formalen Erziehungssystems die Kennzeichen dessen verwirklichen, was man bereits den "neuen Humanismus des elektronischen Zeitalters" genannt hat.

\section{B. Pbilosophische, anthropologische und soziologische Grundlagen für die Pädagogik der Totalen Sprache}

1. Eine Sicht vom Menschen: Jedwede Erziehung gründet sich auf eine philosophische Sicht vom Menschen. Aus dieser Sicht erwachsen nicht nur die Beziehungen, die sich zwischen Erziehern und zu Erziehenden bilden sollen, sondern auch die programmatischen Inhalte und die Übermittlungsformen dieser Inhalte. Mit anderen Worten, man kann sagen, daß die Sicht, die man vom Menschen hat, die allgemeinen Ziele, die besonderen Ziele und die Verfahrensweise einer bestimmten Erziehung festlegt. Man könnte behaupten, daß es so viele mögliche Erziehungsweisen gibt, wie es verschiedene Sichten vom Menschen geben kann.

Die Pädagogik der Totalen Sprache fordert nicht so sehr eine philosophische Sicht vom Menschen als vielmehr die Begegnung mit einem Menschen in konkreter Lage. Ein Mensch von "Fleisch und Blut" verhält sich in seinem Dasein in der Welt zu den anderen Menschen. Er ist ein Mensch in seinem "Hier und Heute", Gestalter seiner Welt und Macher seiner Geschichte. So lehnt man die Idee des Menschen ab und begibt sich auf die Suche nach eben diesem Menschen.

Eine solche Einstellung könnte innerhalb der traditionellen Philosophien bereits als eklektisch gelten. $\mathrm{Da}$ sie gültige Antworten für konkrete Menschen oder auch für eine Mehrzahl von Menschen geben will, würde sie plurale Auffassungen vom Menschen und insofern plurale Philosophien erfordern.

Nun, der Begriff „eklektisch“ ist nicht nur ein Zeichen, das von wortgeprägten, der Wirklichkeit abgewandten Philosophien ausgegeben wird, die den Menschen und die Wahrheit als abgeschlossene Entitäten ansehen, die jedenfalls nichts mit einer Wahrheit und einem Menschen zu tun haben, die nicht sind, sondern ständig werden. 
Wenn in irgendeiner Philosophie diese Pädagogik „eingefangen werden “ könnte, dann wäre es die des verpflichtenden Einsatzes. Eine Philosophie nicht mehr über den Menschen, sondern für den Menschen, wo die Wahrheit nur eine Wahrheit wird, wenn sie die Beziehungen der Einzelmenschen, die mit ihr in Kontakt treten, und der Welt, aus der sie hervorgegangen ist, zu gestalten vermag.

\section{Zusammengefaßt:}

Der Mensch ist Subjekt und nicht Objekt. Deshalb kann er nicht kodifiziert, nicht manipuliert, nicht domestiziert werden, außer wenn er innerhalb seiner eigenen Werte (oder von seiner Wirklichkeitsauffassung her) zu einer kritischen Einstellung und dadurch zur Befreiung befähigt wird.

Der Mensch steht in Beziehung zu den anderen Menschen. Diese Beziehung muß nicht die eines Wettstreits unter Einzelmenschen sein, sondern Beziehung der Zusammenarbeit und der gegenseitigen Achtung. Dazu ist eine dialogische Einstellung nötig. Man sucht, was der Mensch ist, und nicht, was er hat.

Der Mensch lebt in einer Welt, die er gestalten muß. Angesichts seiner eigenen Wahrnehmung und angesichts seiner Reflexion über die Welt richtet er sein Tun aus. Alle einzelnen Teile des Verfahrens münden in eine vollständige, spezifische Wirklichkeit. Ihm müssen konkrete und spezifische Antworten entsprechen.

Da der ganze Mensch und die gesamte Wirklichkeit ein Prozeß sind, ist in jedweder kreativen Handlung dieser Schöpfungsprozeß wichtig. Es gibt keine fertigen Dinge, sondern Dinge in Hinsicht auf das Sein, und Menschen, die in Beziehung zu ihnen mehr werden können.

Kurz gefaßt stellt die Pädagogik der Totalen Sprache die Notwendigkeit dar, mehr das Sein des Menschen zu suchen.

2. Totale Sprache und wechselseitiger Bezug: Die Sprache ist eine Form der Interrelation oder Interaktion. Heute werden nun vielfältige Anstrengungen unternommen, dem Kommunikationsstudium die Aspekte des sinnbildhaften Ausdrucks einzuverleiben, die über die einfach durch linguistische Zeichen übersetzten Aspekte hinausreichen.

Die Pädagogik der Totalen Sprache fordert, über die einfache Interrelation hinauszustreben, die dem reinen Nützlichkeitsaustausch in Masse oder Hörerschaft dient. Sie sucht eine „signifikative Interrelation“ zu schaffen, in der eine Person „signifiziert", Sinn hat für die andere. In diesem Prozeß der Sinngebung kommt nicht nur das "Ich" durch die Entdeckung des „Du“ ans Licht, sondern beide konstituieren das "Wir". Das ist die einzige Beziehungsform, die ein Hervortreten der Person als solcher ermöglicht. Gegenwärtig herrscht gewiß nicht dic Interaktion zwischen den Komponenten unserer Gesellschaft vor, sondern: unsere Gesellschaft ist eine paternalistische, vertikale Gesellschaft, und sie ist es in der Familie, in der politischen Ordnung wie im wirtschaftlichen und im religiösen Bereich.

Wenn es auch eine gewisse soziale Beweglichkeit in unserer straff aufgebauten Gesellschaft gibt, hat doch nur eine Minderheit Zugang zur Erziehung.

Unsere Schule ist eine „mit Mauern umgebene“ Schule mit in sich begrenzten und auf sie selbst ausgerichteten Zielen gewesen. Unsere Schule ist ein Rad innerhalb eines Systems gewesen und hat dieses System noch großartig hochgezuichtet zu einer "gefräßigen Erziehung“, die zwar den Techniker vorbereitet, aber die Person verschlingt. 
Die Pädagogik der Totalen Sprache richtet sich zentral mehr auf die Person als auf das Gesellschaftssystem; sie erarbeitet die Programme, die weniger von einem abstrakten Inhalt ausgehen als vielmehr von einem schöpferischen Kern, da sie die Probleme von der konkreten Wirklichkeit aus stellt, in der der zu Erziehende lebt. So erreicht sie eine ständig stärkere Bewußtwerdung seinerseits. Denn sie richtet seine Erziehung weniger auf die technische Vorbereitung aus, sondern viel stärker auf den Prozeß der Hominisation. Sie entmystifiziert die Technik, verurteilt die Entwicklung um ihrer selbst willen, kritisiert den Konsum. Da diese Pädagogik neue Sinngebungen sucht, läßt sie den Erziehenden neue befreiende Formen in sein Verhalten aufnehmen.

3. Totale Sprache und sozialer Wandel: Sich auf den beschleunigten Wandel zu berufen, den wir erleben, ist ein Gemeinplatz in jeder soziologischen Untersuchung geworden. Aber das typische Phänomen, das sich in unserer Gesellschaft zeigt, ist nicht so sehr das des Wandels an sich, sondern das der unterschiedlichen Geschwindigkeit, mit der der Wandel sowohl auf persönlicher wie vor allem auf institutioneller Ebene eintritt.

Daraus ergibt sich etwas, das wir als „institutionelle Asynchronie“ bezeichnen könnten. In unserer Gesellschaft sind diese Asyndhronien mannigfaltig. Nennen wir nur zwei: Die hochgeschraubten Erziehungserwartungen einerseit und die sehr beschränkte Zahl der Schulen (vor allem gehobeneren Niveaus) andererseits.

Eine "massive Kultur des Bildes und des Tons" auf der einen Seite und eine nicht vorhandene Erziehung, diese neue Sprache zu deuten, wie bislang auch die Unfähigkeit, den Beschauer in einen kritischen und kreativen Beobachter andererseits zu verwandeln.

Diese Asynchronien haben unter uns eine blutarme Gesellschaft geschaffen. Mehr als Normenmangel im etymologischen Wortsinn bindet Passivität, Indifferenz, NichtTeilnahme, Aktionsmangel und Skeptizismus im Umgang mit dem „öffentlichen Anliegen " große Kerne ernüchterter, frustrierter oder einfach rezeptiver Leute. Dieses Problem wird leichthin ignoriert.

Die Pädagogik der Totalen Sprache fördert über ihre Schritte der Perzeption, der Kritik und des kreativen Ausdrucks den sozialen Wandel. Sie fördert authentisch und andererseits gewaltlos den Wandel der Strukturen, wobei nicht einfach ein Element durch ein anderes ersetzt wird (was letztlich kein Wandel ist). Der Wandel wird über einen Prozeß der Personalisierung des zu Erziehenden erreicht. Diese Pädagogik führt über die Personalisierung zu einer neuen Beziehung zwischen den Strukturen, seien sie wirtschaftlich, politisch oder religiös, und bringt von daher einen echten Gesellschaftswandel herauf.

Die Pädagogik der Totalen Sprache ist sowohl auf eine außerschulische oder paraschulische Erziehung wie auf eine systematische Erziehung anwendbar. Sie könnte einige der institutionellen Asynchronien ausgleichen helfen, vor allem die Erziehungsasynchronie zwischen Erwartungen und Möglichkeiten. Sie gebraucht die Massenkommunikationsmittel nicht als einfache Hilfskräfte, sondern als Erziehungsfaktor in Perzeption, Kritik und Kreation. Wenn wir echten Wandel suchen, müssen wir hinnehmen und anerkennen, daß jeder radikale Wandel stets durch die Erziehung beschleunigt worden ist. Die Pädagogik der Totalen Sprache bringt solche Möglichkeit des Wandels mit sich. 


\section{Die Totale Sprache - eine auf die Interrelation gegründete Pädagogik}

Gehen wir davon aus, daß die Sprache eine Gesamtheit artikulierter Laute ist, mit denen der Mensch seine Gedanken und Gefühle ausdrückt, so erfassen wir den Menschen nur in einem einzigen seiner Ausdrucksmittel.

Der Mensch ist Kommunikation, und wenn er es wirksam ist, dienen ihm nicht nur die artikulierten Laute dazu, sich mitzuteilen; so setzt die Pädagogik der Totalen Sprache aus der Erkenntnis, daß sich alles an uns mitteilt und daß der Mensch Kommunikation ist, das ein, was in der Tat wirklich Teil unserer Existenz ist, die Kommunikationsmittel; und sie bleibt nicht bei ihnen stehen, sondern schreitet fort zum Gesamtausdruck des Menschen durch seine Sinne und den angemessenen Gebrauch der Wahrnehmung.

Die Pädagogik der Totalen Sprache erzieht zur Kenntnis der Gesetze der verschiedenen Sprachen, was eine stärkere kritische Haltung gegenüber den Kommunikationsmitteln gestattet.

Die sozialen Kommunikationsmittel bringen unser Leben in Übereinstimmung und domestizieren uns; aber durch sie ist das Erlernen korrekter Denotationen möglich, die zu einer befreienden Erziehung führen und uns einen durch seine umfassende Entwicklung dynamischen Menschen schenken können; darin besteht die ständige Umgestaltung, die die Gesellschaft benötigt zu einem kritischen, schöpferischen und sozialen Menschen.

Darum sieht die Pädagogik der Totalen Sprache unser Verhalten als die Antwort auf die Sinngebungen, die jeder einzelne auf die empfangenen Botschaften vollzogen hat. Diese Sinninhalte bestimmen unser Verhalten und bringen unser Leben, das Leben, das wir leben und leben werden, in Úbereinstimmung.

Die Pädagogik der Totalen Sprache ist eine Antwort, die den neuen Bedürfnissen entgegen kommt, die die "neue Sprache“ des "neuen Menschen“ der heutigen Gesellschaft geschaffen haben.

Dringend muß die heutige Erziehung der Gesellschaft angeglichen werden, von der wir einen Teil bilden und die uns die Gelegenheit gibt, eben diese Gesellschaft zu gestalten.

\section{Ziele der Pädagogik der Totalen Sprache}

$\mathrm{Da}$ die Pädagogik der Totalen Sprache sich auf die schulische wie auf die paraschulische Erziehung erstreckt, suchen die folgenden spezifischen Ziele in allgemeiner Form die Grundziele zusammenzufassen:

$\mathrm{Zu}$ diesem Zwedk haben wir schon die Begriffe Mensch, Gesellschaft, Kommunikation und Erziehung auszumachen gesucht.

Der Mensch ist ein Sein in ständiger Entwicklung, das die Mittel sucht, sich in seiner raumzeitlichen Dimension zu verwirklichen. Dieser Mensch bildet einen Teil der Gesellschaft, und diese ist eine Gesamtheit von Menschen, die auf der Suche nach einem gemeinsamen Wohl untereinander handeln. Diese Gesellschaft macht eine Kommunikation möglich, die uns zur Erziehung, das heißt, zu einem intern befreienden und kreativen Einsatz in unserer sozialen Umwelt bringen kann. 
Hier ist klarzustellen, daß wir in schulischer Hinsicht die Trennung zwischen dem Alltagsleben, in dem der Schüler intensiv die Gegenwart erlebt, und dem schulischen Leben, das ihn in eine Welt der Vergangenheit versetzt, zu vermeiden suchen.

Als von dieser Pädagogik abgeleitet erweisen sich die folgenden spezifischen Ziele:

1. Die sozialen Kommunikationsmittel nicht nur in Instrumente der Information, sondern in echt bildende Kräfte umzuwandeln.

2. In die neue Kultur die Personen zu integrieren, die die sozialen Kommunikationsmittel hervorgebracht haben.

3. Teilnahme, Verstehen und Dialog durch die Perzeption und die Reflexion zustande zu bringen.

4. Situationen zu schaffen, in denen die Person ihre Kreativität als Folge der neuen Ausdrucksformen und der neuen Erziehungsinhalte bekundet.

5. Den Menschen für eine Begegnung mit der Problematik seiner Umwelt empfindsam zu machen, indem man seine persönliche Teilnahme als Einsatz in seiner Wirklichkeit fördert.

6. Den Menschen zu befähigen, die Zeichen, die ihn erreichen, ganzheitlich wahrzunehmen, und die Struktur, die sie bedingt, zu verstehen.

7. $\mathrm{Zu}$ erreichen, daß die Person die neuen Sprachen der sozialen Kommunikationsmittel versteht, gebraucht und beherrscht, indem man sie in die Inhalte der neuen Kultur integriert.

8. Zum angemessenen Gebrauch der sozialen Kommunikationsmittel zu erziehen, indem man den Konsum der Kommunikation in passiver Form vermeidet.

9. Die Bewußtseinsbildung zu begrüßen, daß die sozialen Kommunikationsmittel Vermittler der Annäherung und des Zusammenlebens sein müssen.

10. Im menschlichen Sein die Fähigkeit zum Dialog innerhalb eines sozialisierenden Rahmens zu fördern.

11. Die Befreiung des Menschen durch die Entwicklung seiner Kreativität angesichts seiner gegenwärtigen Situation zu erreichen, die durch die Kommunikationsmittel of tmals entstellt wird.

12. Eine Person heranzubilden, die fähig ist, sich ständig und dauerhaft dem stetigen Wandel anzupassen.

13. $\mathrm{Zu}$ hoffen, daß künftig die Schule als solche verschwindet, so daß die gesamte Gesellschaft die "Schule" wird und alle ihre Mitglieder zugleich zu Erziehende 14. Dem Menschen seine Mythen (Sinninhalte) und ebenso seine Riten (Verhalten) bewußt zu machen, damit er sich durch diese Begegnung mit sich selbst als Person in einer Gemeinschaft verwirklicht.

15. $\mathrm{Zu}$ erreichen, daß der in die Gemeinschaft integrierte Mensch die Verwirklichung des Gemeinwohls sucht. 


\section{Die Methodologie der Totalen Sprache}

Methode ist ein systematisches Maß, ein Weg, dem man folgt, um ein bestimmtes Ziel zu erreichen. Die Pädagogik der Totalen Sprache hat ihre eigenen Methoden. Gegenstand der Methoden der Pädagogik der Totalen Sprache ist die Erziehung zur Befreiung; einen Menschen heranzubilden, der sich in seinem eigenen kritischen, schöpferischen und sozialisierenden Tun in der dauernden Neustrukturierung der Gesellschaft einsetzt.

Die Methodologie der Totalen Sprache befaßt sich mit gewissen Grundfundamenten, die herauszuheben sie interessiert ist, um so die charakteristischen Linien der Methodologie offenzulegen. Diese Prinzipien sind: A. Die Interrelation oder die dialogische Kommunikation; B. der schöpferische Kern; C. die geeignete Verwendung der verschiedensten Ausdruckstechniken; D. Kennzeichen eines Erziehungszentrums, das mit der Methodologie der Totalen Sprache arbeitet.

\section{A. Interrelation oder dialogische Kommunikation}

Die Erziehung ist Kommunikation. Ohne Kommunikation kann es keine wahre Erziehung geben. Gerade das schwerwiegendste Problem des traditionellen Schulsystems ist das Fehlen der Kommunikation. Alle menschliche und soziale Veränderung ist eine Folge der Dialoghaltung. Darum glauben wir, daß die horizontale oder teilnehmende Kommunikation der einzige Prozeß ist, der den Menschen als Menschen verwirklichen und ihn zur Schöpfung und zum Einsatz bringen kann. Um diese dynamische Situation ständiger Entwicklung zu ermöglichen, ist die dialogische Kommunikation, die das kritische Denken schafft, unumgänglich. Innerhalb des erzieherischen Prozesses kann die Kommunikation nicht auf ein einfaches Weitergeben von Daten (der Information) beschränkt bleiben, sondern ist vielmehr ein Prozeß der Entdeckung unserer selbst und der Welt, die uns vermittels des Dialogs umgibt. Aufgrund dieses Grundsatzes können die Probleme, die die moderne Kommunikation den Erziehern aufgibt, nicht auf eine bessere oder schnellere Úbermittlung der Kenntnis beschränkt bleiben. In einer Welt mit einer so beängstigenden Vorherrschaft der informativen Massenmedien ist es nicht befremdend, daß die informierende Schule in eine tiefe existentielle Krise geführt hat.

Die Kommunikationsmittel bestimmen das Verhalten des Menschen von heute, formieren und konformieren sein Leben: darum sind die Methoden der Pädagogik der Totalen Sprache die eines jeden Kommunikationsmittels. Sie sind ausgerichtet durch die strukturalistische Semiotik jedes einzelnen und eine Psychologie, die nicht-direktiv auftritt.

Von dieser Wirklichkeit der sozialen Kommunikationsmittel aus umfaßt die Totale Sprache die Erziehung in allen vorhandenen Kommunikationsarten.

Die kinetische Kommunikation ist die Sprache der Aktion. Innerhalb der Methodologie der Totalen Sprache muß dieser Kommunikationstypus von den ersten Lebensjahren des Kindes an stark betont werden. Der Rhythmus, der Tanz, die Mimik, die Bewegungserziehung werden die angemessensten Mittel für sie sein.

Die orale Kommunikation muß das Kind, den jungen Menschen, den Erwachsenen dazu bringen, „sein Wort“ zu sagen. Das wird nur möglich sein in einem Klima der 
Horizontalität (einer nicht-direktiven Psychologie), in der der Erzieher belebt, motiviert, und den Erziehungsprozeß stimuliert.

Uber diese herzliche Beziehung zwischen Lehrer und Schüler hinaus ist es weniger wichtig, die mündliche Kommunikation, den Kontakt der Schüler untereinander zu fördern. Der Dialog geschieht spontan, wenn es eine gemeinsame Sache gibt, die ihn motiviert, und falls eine günstige Umwelt durch eine gut angewandte Gruppendynamik geschaffen wird.

Wir leben heute in einer Bild-Kultur. Der Weg, der die zu Erziehenden zu Kenntnis und Überzeugung bringt, ist weit mehr das Bild als der Text. Die Abstraktionen, die Symbole treten ihre Stelle den Wirklichkeiten oder ihrer konkreten Darstellung ab. Die Kommunikation in unserer Gesellschaft kann sich nicht auf den Gebrauch der stereotypen Zeichen beschränken, sondern muß sich fundamental auf das Bild gründen. Das Aufnehmen dieser Kommunikationen und das Wissen, sich mit ihrer Hilfe auszudrücken, ist ein Aspekt von erstrangiger Wichtigkeit in der Methodologie der Totalen Sprache.

Der angemessene Gebrauch dieser neuen Sprachen wird es ermöglichen, daß der Schüler vom Status des bloßen Rezipienten zum aktiven Perzeptoren und damit zum kommunikativen Schöpfer wird, was allerdings nur in einem dauerhaften und ununterbrochenen Feedback geschehen kann.

Diese Methodologie des Kommunikationsprozesses gründet sich auf die reflexive Denotation, die zur Kreativität führt. Die Denotation und damit die kritische Reflexion, die nicht einmündet in die Aktion, verwertet den Kommunikationsprozeß nicht, sondern schränkt ihn auf die Weitergabe von Kenntnissen ein. Darum ist die dialogische Kommunikation der einzige Weg, der zur Teilnahme des zu Erziehenden am Lernprozeß führt.

Die dialogische Kommunikation schließt als methodologischen Schritt die Gruppendynamik ein. Die Gruppendynamik umfaßt volle Teilnahme und macht Freiheit und persönlichen Einsatz aller Gruppenkomponenten gemeinsam. Nur unter diesen Bedingungen kann absolut vollständige erzieherische Interrelation erreicht werden.

Ein so begriffener und durchgehaltener Typus der Dynamik ist wie ein lebendiges Kommunikationslabor, das von sich aus die angemessenste Praxis darstellt, um kritisch den Mitteln der Masseninformation zu begegnen.

Um diese Ziele der Gruppendynamik zu erreichen, ist es unerläßlich:

a) Sich in seiner Ganzheit angenommen, integriert und gewertet $\mathrm{zu}$ verstehen und $\mathrm{zu}$ fühlen von jenen, mit denen man vereint ist. Das nennt man die Einschlußnotwendigkeit.

b) Sich voll verantwortlich zu fühlen für das, was die Gruppe ist: für ihre Strukturen, ihre Aktivitäten, ihr Wachstum und ihre Auszeichnung. Das nennt man die Notwendigkeit der Verantwortung.

\section{B. Schöpferischer Kern}

Der schöpferische Kern ist die zweite Bedingung, damit die Methodologie funktionieren kann. Er ist das aus einer spezifischen menschlichen Gruppe aufgestiegene Interessenszentrum, das normalerweise seinen Ursprung in einem Mittel der sozialen Kommunikation hat, in einer Zeitschrift, Erzählung, Veröffentlichung, Zeitung, 
Schallplatte, einem Radioprogramm, Fernsehprogramm, Film usw., deren Zweckbestimmung es ist, die Erziehungsziele der betreffenden menschlichen Gruppe zu verwirklichen.

Jeder Kern muß der Gruppe Probleme stellen. Im gegenteiligen Fall würde er die dieser Methodologie eigenen Ziele nicht erfüllen.

Um die programmatischen Ziele mit den Interessen und Bedürfnissen der Erziehungsgemeinschaft zu synchronisieren, müssen die schöpferischen Kerne aus der soziokulturellen Struktur resultieren.

Der schöpferische Kern entwickelt sich in zwei grundlegenden methodologischen Momenten.

1. Analytisches Moment: Dessen Zweckbestimmung ist die Erziehung zur Perzeptivität, zur Intuition und zur kritischen Uberlegung, dank der Entwicklung der folgenden drei Phasen:

a) Denotatives oder formales und objektives Lesen der im Kern vorgelegten Zeichen. Diese Phase setzt die Kenntnis der verschiedenen Semiotiken voraus. Ihr Ziel ist es, in möglichst erschöpfender Form das Wesen und die Bedeutung der Zeichen zu erfassen.

b) Konnotatives Lesen, das zum subjektiven Ausdruck dessen führt, was jedes Zeichen dem Aufnehmenden innerhalb seines Rahmens persönlicher und sozialer Konditionierungen nahelegt. Auch der Autor und Schöpfer des Zeichens ist mit seinen eigenen Konnotationen, die er zu entdecken bemüht sein muß, belastet.

c) Strukturales Lesen geschieht, wenn man zwischen dem denotativen und konnotativen Lesen die Beziehung herstellt, indem man die Bezogenheit der Elemente berücksichtigt. Das setzt die Kenntnis des persönlichen Gesetzes des Autors voraus, wie auch die kultur-historische Struktur, in der er zuhause ist.

2. Synthetisches Moment: Dieses ist darauf ausgerichtet, die Kreativität des zu Erziehenden zu bilden. Die Entwicklung des Kerns erzeugt die Schaffung motivierter Unterkerne im perzeptiven Prozeß, der seinen Höhepunkt findet mit der Schaffung neuer Sinngehalte. Der Prozeß der Kommunikation ist das Moment, in dem der zu Erziehende vom Status des Perzeptors zu dem des Kommunikators gelangt.

\section{Ausdruckstechniken}

Ein anderes Kennzeichen der Methodologie ist die Schaffung von Ausdrucksformen oder -techniken, die es dem zu Erziehenden ermöglichen, seine Überzeugungen durch eigene Zeichen mitzuteilen.

Die Schaffung von Zeichen wird möglich dank dem Lernprozeß der verschiedenen Semiotiken, besonders derer, die in den sozialen Kommunikationsmitteln verwendet werden.

Die am allgemeinsten gebrauchten Techniken in der Methodologie der Totalen Sprache sind folgende:

1. Bei den kinetischen Zeichen: Mimik, Pantomime, Tanz, Ballett und weitere kinetische Zeichen, die, ob kodifiniert oder nicht, für den Menschen und ganz besonders für das Kind bedeutsame Formen der Rationalität darstellen. 
2. Bei linguistischen Zeichen (mündlich und geschrieben); sowohl die Zeichen der gesprochenen Sprachen wie die Schriften stellen weitere machtvolle Ausdruckstechniken dar: Monologe, Dialoge, Interviews, Rundgespräche, Reportagen, Slogans, geschriebene Sätze, explikative Texte, ergründende Texte, abgefaßt in den unterschiedlichsten Formen, Rede, Theater, Poesie usw.

3. Bei Bild-Zeichen: Dieser Teil umschließt eine große Anzahl in Bildern grundgelegte Semiotiken: Photographie, Zeichnung, Skulptur, Mosaik, Bildfenster usw.

4. Bei tönenden Zeichen (Worten mit Tönen): Gesänge, Klangmontagen, Kassetten, Radioaufzeichnungen verschiedener Art.

5. Bei Worten mit Bildern: Die Vereinigung der traditionellen Sprache (mündlich und geschrieben) mit dem Bild-Zeichen läßt eine Reihe weithin in der Methodologie der Totalen Sprache verwendeter Ausdruckstechniken entstehen. Einige Beispiele: Anzeigenseite, Seite in Zeitungen und Magazinen, illustrierte Kurzgeschichten, Erzählungen (comics) in Photomontage, Photonovellen, Plakate, Anschläge usw.

6. Bei Worten mit Bild-Zeichen und Klangzeichen: Diese Gruppe bereichert uns mit den Ausdruckstechniken, die für die gegenwärtige Kommunikation recht bezeichnend sind: Fernsehen, Film, Videorekorder.

\section{Kennzeichen eines Erziehungszentrums, das mit der Methodologie der Totalen Sprache arbeitet}

1. Nichtsystematisches Zentrum: Ein erstes Kennzeichen ist seine unsystematische Anlage. Das bedeutet eine tiefe Veränderung in den wechselseitigen erzieherischen $\mathrm{Be}-$ ziehungen. Es setzt voraus, daß das Zentrum:

a) nicht einem akademischen Lehrplan zentraler Stellen unterworfen ist. Sowohl die programmatischen Inhalte wie die Ausdrucksformen für diese müssen Ergebnis wechselseitigen Austausches bei Sicht und Suche der Erziehungsgemeinschaft nach ihrer eigenen Wirklichkeit sein. Die schöpferischen Kerne müssen deshalb aus der eigenen Gemeinschaft hervorgehen.

Dieses setzt Arbeitsmöglichkeit voraus:

- ohne starre und vorher festgelegte Stundenpläne;

- ohne stereotype Planungen;

- ohne mathematische Bewertungen;

- ohne äußere und verpflichtende Disziplin.

b) Hingegen müssen die wechselseitigen Beziehungen, die sowohl aus der Gruppendynamik wie aus dem schöpferischen Kern entstanden sind, förderlich sein für:

- eine Umwelt der Verantwortung, der Teilnahme, der Aufrichtigkeit und der Freiheit $\mathrm{zwischen}$ den verschiedenen Mitgliedern der Erziehungsgemeinschaft;

- die Entwicklung der eigenen schöpferischen Fähigkeiten;

- die Möglichkeit, Lösungen für die eigenen Probleme in der Praxis zu suchen;

- Verantwortung für alle Mitglieder der Gemeinschaft beim Suchen nach neuen Quellen der Erfahrung und der Erkenntnis. 


\section{Wechselseitige Bezogenbeit der Elemente der Erziebungsstruktur}

Zwischen den Elementen (Schülern, Erziehern, Familienvätern eines Erziehungszentrums, das mit der Pädagogik der Totalen Sprache arbeitet) wird ein ständiger wechselseitiger Dialog bestehen.

Er muß eingebunden sein in:

- gemeinsames Suchen;

- Entdeckung, Wertung und Kritik;

- Schöpfung.

Der Schritt von einer traditionellen Struktur zu einer anderen dynamischen Struktur stïtzt sich auf die dialogischen wechselseitigen, horizontalen und teilhabenden Beziehungen zwischen all ihren Elementen. Bei diesem Strukturtypus kann man folglich nicht die vertikale Autorität aufrechterhalten. Das bringt mit sich, daß der Direktor und die anderen "Autoritäten " des Zentrums wie alle anderen Mitglieder der Gruppe an der delegierten Autorität teilnehmen.

In derselben Weise fühlen sich die Mitglieder der Verwaltungsmannschaft, die sich der Erziehungsgemeinschaft dialogisch integrieren, für den Erziehungsprozeß voll verantwortlich.

\section{Kernbildung}

Damit die Pädagogik der Totalen Sprache wirksam arbeitet, muß die Erziehungsgemeinschaft ein konvergierendes Zentrum sein, zusammengesetzt aus den Erziehern, den zu Erziehenden, Familienvätern und anderen Mitgliedern des Ortes oder der Bevölkerungsgruppe, wo das ,schulische "Zentrum sich befindet.

- Nur in der Praxis wird die wechselseitige Bezogenheit der gesamten Bevölkerungsgruppe erreicht. In einem zweiten Schritt wird man die wechselseitige Bezogenheit zu anderen Gemeinschaften suchen.

- Die Gruppierung der Schüler in der Erziehungsgemeinschaft geschieht nach Interessen, Bedürfnissen und Kenntnissen.

- Für jede eine dieser Gruppen ist ein dialogischer Erziehungskoordinator mit seiner vollen Zeit verfügbar.

- Die Erziehungskoordinatoren bilden eine tief vereinte und verantwortliche Mannschaft. Auf sie stützt sich der gute Ablauf des Erziehungsvorgangs.

- Die Familienväter müssen gleichfalls voll integriert werden und ihnen kommt es zu, gemeinsam mit den Erziehungskoordinatoren die Unruhen und Interessen der zu Erziehenden anzustoßen und zu verursachen.

- Die Planung muß flexibel und dynamisch sein und den Interessen der Gemeinschaft entsprechen, ohne die Kreativität der zu Erziehenden zu beeinträchtigen.

- Um die Kernbildung zu erreichen, ist es unumgänglich, daß die äußere Anlage des Erziehungszentrums Tag und Nacht von den verschiedenen Mitgliedern des Gemeinschaftskerns benutzt werden kann. Diese paraschulische Aufgabe geschieht mit der gleichen Methodologie der Totalen Sprache.

\section{Erziehungsarbeit zentriert auf den schöpferischen Kern}

Die Schüler erhalten die Information über:

a) das Wesen (das Hier und Heute der Erziehungsgemeinschaft); 
b) über die Mittel der sozialen Kommunikation (Bücher, Zeitungen, Zeitschriften, Erzählungen, Radio, Schallplatten, Fernsehen, Filme usw.).

Die Interessen, Entdeckungen, Motivationen, Erfahrungen und Lebensumstände der Schüler gegenüber diesen Interessenszentren stoßen die schöpferischen Kerne an, die ihrerseits zu Gebieten der Erkenntnis, der Reflexion und der Kreativität werden.

Der Kern bringt die Schaffung von Zeichen (wichtigster Akt der Erziehungsaufgabe). Die Entwicklung des Kerns kann einem von dem Kern selbst und durch die Interessen der Schüler nicht motivierten Stundenplan nicht unterworfen werden.

Als Endschritt werden die kreativen Aktivitäten und die Aktivitäten der Forschung für jede Arbeitsgruppe, die so zu einer Verallgemeinerung der Gebietserkenntnis gelangt, gemeinsam zusammengestellt.

Über die durch die Schüler entdeckten Interessen hinaus werden die dialogischen Erziehungskoordinatoren andere Interessenszentren vorstellen, die die Schüler zur Entdeckung neuer Werte führen.

Innerhalb des Erziehungsprozesses wird man der Erziehung der Muße und der Freizeit Bedeutung beimessen.

\section{S UM M A R Y}

The concept of "total language" was created by the French Marist Antoine Vallet. Total language aims at giving a new relation to human life and modern communication. The concept was received with special interest in Latin America. Francisco Gutiérrez Pérez, F.S.C., introduced Total Language Courses in Peru for the first time in 1969. Today the method is known in more Latin American countries. In 1973 new courses begin in Quito, Lima and Santiago. Total language tries to bring all the means and possibilities of communications into practical and theoretical education. English Text on the subject published by Multimedia International, Rome (Volume 5: "Total Language" a new approache to education).

\section{RÉSUMÉ}

La dénomination de "langue totale" qu'il faut associer au nom d'Antoine Vallet, frère mariste français, veut donner une orientation de base qui crée un rapport nouveau entre l'école et la vie et incorpore en vérité pleinement le monde des moyens de communication modernes. Ce projet a trouvé un intérêt particulier en Amérique Latine. Francisco Gutiérrez Pérez FSC introduisit pour la première fois en 1969 de cours de "langue totale“ au Pérou. Aujourd'hui, on les connait déjà dans beaucoup de pays d'Amérique Latine. En 1973 commencent de nouveaux cours de perfectionnement à Quito, Lima et Santiago. La langue totale introduit toutes les marques des communications existantes dans l'éducation pratique comme dans la pédagogie théorique.

\section{R E S U M E N}

La expresión "lenguaje total“, acuñada por el Hermano Marista francés Antoine Vallet, indica una orientación fundamental que sitúa la escuela y la vida en una nueva relación, a saber: aprovechar plenamente el mundo de los modernos medios de comunicación social. Esta orientación suscitó un interés especial en Latinoamérica. Francisco Gutiérrez Pérez FSC dió por primera vez en Perú cursos de "lenguaje total“ en 1969. Hoy son ya conocidos en muchos paises latinoamericanos. En 1973 comienzan nuevos cursos de promoción en Quito, Lima y Santiago de Chile. El "lenguaje total“ aporta expresiones comunes de los actuales medios de comunicación social tanto a la educación práctica como a la pedagogía teórica. 